\title{
ORIGINAL ARTICLE Role of the ventral striatum in developing anorexia nervosa
}

\author{
A-K Fladung ${ }^{1}$, UME Schulze ${ }^{2}$, F Schöll ${ }^{1}, K_{\text {Bauer }}{ }^{1}$ and G Grön ${ }^{1}$
}

Functional imaging data in adult patients with anorexia nervosa (AN) support a dysfunctional signal in the ventral striatum as neural signature of AN. In the present study, development of this signal was investigated with the prediction that a characteristic pattern of ventral-striatal signalling will be shown in response to cues associated with food restriction that reflects the evolvement of starvation dependence over time. The signal was assessed in adolescent patients with AN, whose duration of illness was about five times shorter relative to the adult sample. During functional magnetic resonance imaging subjects were required to estimate weights of body images (underweight, normal weight, overweight) and to process each stimulus in a self-referring way. Relative to age-matched, young healthy controls, underweight stimuli were already associated with greater activity of the ventral striatum, and processing of normal-weight stimuli elicited already reduced signalling. Subjective preferences showed exactly the same pattern of results. Relative to adult AN, the present data reveal a developing dysfunctional signal that, if untreated, will essentially contribute to the maintenance of AN. We discuss putative mechanisms that may play a crucial role in the development of $\mathrm{AN}$, and also deduce new hypotheses about the involvement of the midbrain dopamine system, of which illness-related alterations may contribute to the development of AN.

Translational Psychiatry (2013) 3, e315; doi:10.1038/tp.2013.88; published online 22 October 2013

Keywords: anorexia nervosa; fMRl; pathogenesis; reward; ventral striatum

\section{INTRODUCTION}

Anorexia nervosa (AN) usually develops around the onset of puberty. If untreated, the illness can become chronic and interfere with the course of normal development. There is a negative relation between illness duration and treatment outcome, and hence treatment is needed as early as possible. ${ }^{1}$ Systematic identification of the early eating-disorder symptoms of AN reveals a subgroup of patients characterized by excessive exercise and extreme eating-disorder attitudes. ${ }^{2}$ This aligns with 'activity-based' animal models, where diet and hyperactivity were suggested to contribute to the onset and maintenance of AN via involvement of the ventral striatum. ${ }^{3}$ As part of the mesolimbic dopaminergic system the ventral striatum is crucially involved in the processing of reward and motivational salience. ${ }^{4-9}$ In animal models conditioned activity in this system was associated with increased self-starvation, ${ }^{10}$ emphasizing the addictive quality of AN as starvation dependence, much like drug dependence. ${ }^{11}$

Based on these predictions we have recently shown that adult AN is associated with signal changes in the ventro-striatal reward/ motivation system. Compared with the healthy women (comparison group), patients showed increased neural signalling upon stimuli associated with food restriction and reduced signalling upon normal-weight stimuli. ${ }^{12}$

In the present study we investigated the pathogenesis of this signal change and predicted that if AN represents the features of a dependence disorder the characteristic pattern of ventral-striatal signalling should evolve over time. To this end we examined a group of adolescent AN patients, whose disease duration was about five times shorter than that of the previously described adult sample (see Table 1). Patients were confronted with cues associated with food restriction to different degrees (underweight, normal-weight and overweight female body stimuli). During functional magnetic resonance imaging (fMRI) participants were asked to rate the weightings of the stimuli ('weight' task) and to rate the stimuli self-referentially ('feel' task).

\section{MATERIALS AND METHODS}

Thirteen adolescent girls who met the criteria for AN according to the Diagnostic and Statistical Manual of Mental Disorders ${ }^{13}$ were recruited from the Department of Child and Adolescent Psychiatry/ Psychotherapy at UIm University. Patients with an actual body mass index (BMI) lower than or equal to the 10th percentile with reference to a study on 17275 girls were included. ${ }^{14}$ Cognitive-emotional symptoms were rated via parent interviews, self-report and questionnaire data (Eating Disorder Inventory; Eating Disorder Examination Questionnaire). ${ }^{15,16}$ Ten patients met the criteria for the restrictive subtype. For three patients engagement in binge eating/purging behaviour could not be entirely ruled out. None of the patients had a lifetime diagnosis of bulimia nervosa. One patient received a selective serotonin reuptake inhibitor (fluoxetine-20 mg per day) and an atypical antipsychotic medication (quetiapine-200 mg per day). All other participants were medication-free. Exclusion criteria were substance abuse or dependence, suicidality, epilepsy, cardiovascular disease, pheochromocytoma and hyperthyroidism.

Fourteen healthy controls $(\mathrm{HC})$ were recruited from public schools. They had no lifetime or concurrent diagnosis of any psychiatric disorder or medical illness.

All participants and their parents or caregivers gave written informed consent after a complete and detailed description of the study. All methods and procedures were approved by the ethics committee of the University of Ulm and conformed to the Code of Ethics of the World Medical Association (Declaration of Helsinki).

Patients and control subjects did not differ on age and educational level (college preparatory high school 'Gymnasium', AN $n=10$; HC $n=11$; Junior High School 'Realschule', AN $n=3, \mathrm{HC} n=3$ ). Patients had a significantly

\footnotetext{
${ }^{1}$ Department of Psychiatry and Psychotherapy, University of Ulm, Ulm, Germany and ${ }^{2}$ Department of Child and Adolescent Psychiatry/Psychotherapy, University of Ulm, Ulm, Germany. Correspondence: Professor G Grön, Department of Psychiatry and Psychotherapy, Section of Neuropsychology and Functional Imaging, University of Ulm, Leimgrubenweg 12-14, 89075 Ulm, Germany.

E-mail: georg.groen@uni-ulm.de

Received 24 July 2013; revised 5 September 2013; accepted 8 September 2013
} 
Table 1. Demographic and clinical variables for adolescents with and without diagnosis of anorexia nervosa

\begin{tabular}{|c|c|c|c|c|c|c|c|}
\hline \multirow[b]{2}{*}{ Variable } & \multicolumn{2}{|c|}{ Healthy controls $(\mathrm{N}=14)$} & \multicolumn{2}{|c|}{ Anorexia nervosa $(\mathrm{N}=13)$} & \multicolumn{3}{|c|}{ Analysis } \\
\hline & Mean & s.d. & Mean & s.d. & $\mathrm{t}$ & $d f$ & $P$ \\
\hline Age (years) & 16.57 & 1.09 & 16.0 & 1.08 & -1.37 & 25 & 0.184 \\
\hline Years of education & 10.64 & 1.01 & 9.93 & 1.12 & -1.76 & 25 & 0.090 \\
\hline $\mathrm{BMI}\left(\mathrm{kg} \mathrm{m}^{-2}\right)$ & 20.8 & 1.9 & 16.6 & 1.2 & 6.89 & 25 & $<0.001$ \\
\hline Age at onset (years) & - & - & 14.89 & 1.54 & - & - & - \\
\hline Duration of illness (months) & - & - & 13.66 & 15.9 & - & - & - \\
\hline EDI 2 & 178.64 & 29.27 & 254.54 & 55.47 & 4.49 & 25 & $<0.001$ \\
\hline Drive for thinness & 10.10 & 2.30 & 23.77 & 9.51 & 5.23 & 25 & $<0.001$ \\
\hline Bulimia & 10.00 & 2.39 & 11.69 & 4.75 & 1.18 & 25 & 0.248 \\
\hline Body dissatisfaction & 18.0 & 4.76 & 29.20 & 10.64 & 3.56 & 25 & 0.002 \\
\hline EDE-Q & 0.16 & 0.18 & 1.95 & 1.46 & 4.54 & 25 & $<0.001$ \\
\hline Weight concern & 0.11 & 0.19 & 2.11 & 1.72 & 4.32 & 25 & $<0.001$ \\
\hline Shape concern & 0.34 & 0.33 & 2.58 & 1.75 & 4.69 & 25 & $<0.001$ \\
\hline
\end{tabular}

Abbreviations: BMI, body mass index; EDI 2, Eating Disorder Examination, total value and three subscales; EDE-Q, Eating Disorder Examination - Questionnaire, total value and two subscales.

lower actual BMI compared with controls. Patients scored higher on selfratings concerning eating disorder pathology, especially on drive for thinness, body dissatisfaction, and weight and shape concerns. No difference was found concerning bulimic symptoms, reflecting the predominant presence of the restrictive subtype of AN in the sample (see Table 1).

\section{Paradigm}

As in our previous study, ${ }^{12}$ stimuli were 48 computer-generated nude female images of the same woman (height: $165 \mathrm{~cm}$ ), varying in BMI and body posture, with $15^{\circ}, 25^{\circ}, 35^{\circ}$ or $45^{\circ}$ deviation from the centered vertical body axis to the left or right. Sixteen images each were repeated three times in one of three weight categories: underweight (BMl: $11 \mathrm{~kg} \mathrm{~m}^{-2}$ ), normal weight (BMl: $19-22 \mathrm{~kg} \mathrm{~m}^{-2}$ ) and overweight (BMl: $30 \mathrm{~kg} \mathrm{~m}^{-2}$ ). Photorealistic textures gave a familiar presentation of specific features according to the particular BMI. In the task referred to as 'feel', subjects processed each stimulus in a self-referring way ('Imagine you have the same body shape as this woman. How would you feel?'), giving ratings of each stimulus using four buttons on a keyboard (1 ('very bad') to 4 ('very

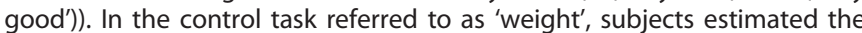
weight of each body stimulus by using one out of the four keyboard buttons according to four weight categories (30, 45, 60 and $75 \mathrm{~kg}$ ). The entire session comprised 72 'feel' and 72 'weight' tasks presented in pseudo-randomized order, such that any given task did not appear more frequent than two times in direct succession. Each trial started with a blank screen (average duration: $996 \mathrm{~ms}$ ), followed by presentation of the stimulus together with one of two scales indicating the task ('feel' or 'weight'). Each stimulus was presented until button press (max. $3500 \mathrm{~ms}$ ), followed by a blank screen (trial duration: 4000-6000 ms). Seventytwo additional null trials (blank screen for 4000-6000 ms) were randomly interspersed. Stimuli were presented via liquid crystal display video goggles (Resonance Technologies, Northridge, CA, USA).

\section{Scanning procedures}

T2*-sensitive gradient echo echo-planar imaging was performed on a 3-Tesla MAGNETOM Allegra (Siemens, Erlangen, Germany). Twentyseven transversal slices were acquired with a repetition time of 2000 ms, echo time of $39 \mathrm{~ms}$ and a receiver bandwidth of $3905 \mathrm{~Hz}$ per pixel in ascending direction (matrix $64 \times 64$ pixels, pixel size $3.6 \times 3.6 \mathrm{~mm}^{2}$, slice thickness $3 \mathrm{~mm}$, with $1.0 \mathrm{~mm}$ gap). Because of randomly assigned jitter times experimental sessions could comprise acquisition of 519-531 images. The first 4 volumes were discarded because of $\mathrm{T} 1$ equilibration effects. An individual sagittal MPRAGE T1 structural image was acquired (matrix $256 \times 256$ pixels, voxel size $1 \times 1 \times 1 \mathrm{~mm}^{3}$ ).

\section{$f M R I$ analysis}

Data analysis was performed using SPM8 (Release 4290, http://www.fil.ion. ucl.ac.uk/spm). fMRI data series underwent slice-time correction and spatial realignment. Individual T1 images were co-registered onto the individual average echo-planar imaging from the realigned time series. Normalization of fMRI images into canonical MNI (Montreal Neurological Institute) space was achieved using the DARTEL process stream. ${ }^{17}$ Volumes were resliced to a voxel size of $2 \times 2 \times 2 \mathrm{~mm}^{3}$ and spatially smoothed using an $8 \mathrm{~mm}$ fullwidth at half-maximum Gaussian kernel.

Design matrices of individual general linear models incorporated six regressors that combined the factor stimulus (underweight, normal weight, overweight) with the factor task ('feel', 'weight'), with onsets at the time of appearance of the corresponding event and convolved with the canonical hemodynamic response function. Group analysis was performed using analysis of variance, with one between-subjects factor and one within-subject factor reflecting combinations of task by stimulus. A factor, 'subject', was added to the design matrix in order to remove variability as a result of differences in the participants' average responses.

Statistical analyses of group-by-condition interactions were constrained to the bilateral ventral striatum using the PickAtlas software (Version 2.3, Wake Forest University School of Medicine, Winston-Salem, NC, USA). ${ }^{18,19} \mathrm{~A}$ whole-brain analysis was performed to search for statistical effects beyond the a priori defined region of interest (ROI). As nominal level of significance for group-by-stimulus interactions, a voxelwise threshold of $P<0.05$, with family-wise error (FWE) correction for multiple comparisons, was used for the ROI-based and whole brain analysis. Additionally, an extended ROIbased analysis with a more lenient threshold compared with the main analysis was conducted to test for effects in other brain regions processing incentive motivation and reward. From an automated meta-analysis of 329 studies loading highly on the feature 'reward' (http://www.neurosynth.org/ features/reward) an inclusive mask was prepared within which the effect of the group-by-stimulus interaction contrast was tested at a statistical threshold of $P<0.005$, uncorrected, in combination with a cluster extent of at least 10 contiguously significant voxels (for details, see Supplementary Information).

Only group-by-stimulus interactions and post-hoc within-group differences were considered relevant for inference of statistically reliable effects. Explorative post-hoc tests of between-group differences were computed, although these may be confounded by unspecific differences of activation magnitude in both groups. Using the normal-weight stimulus as a reference, two group-by-stimulus interactions specified for both tasks 'feel' and 'weight' were assessed: the effect of the underweight body stimulus versus the normal-weight body stimulus and the effect of the overweight body stimulus versus the normal-weight body stimulus. Inspection of the height of effect sizes for each of the resulting four tests was used to evaluate which of the group-by-stimulus interactions contributed to the overall significance (see Supplementary Information).

\section{RESULTS}

Behavioral data

A repeated-measures analysis of variance for rating scores from the 'weight' and 'feel' tasks revealed a significant interaction of all 
main-factors group, task and stimulus $(F(2,50)=17.04, P<0.0001$; Figure 1). Bonferroni post-hoc tests (nominal alpha-level $P<0.05$ ) revealed no significant between-group differences for 'weight' task ratings of underweight, normal-weight and overweight stimuli (for all $P$ values $=1.00$ ). Differences were significant in the 'feel' task. AN patients had higher positive scores relative to $\mathrm{HC}$ for the underweight stimuli $(P<0.001)$ but gave underweight stimuli the same preference compared with normal-weight stimuli $(P=1.00)$. HC preference for normal-weight images was significantly higher when compared with patients $(P<0.001)$ and controls' ratings of normal weight differed significantly from their rating of underweight $(P<0.001)$. Both groups did not differ on overweight ratings $(P=1.00)$

\section{fMRI data}

An F-test evaluated the two relevant group-by-stimulus interactions for the 'feel' and 'weight' tasks. This yielded a significant effect in the bilateral ventral-striatal ROI (right: maximum peak at $x, y, z$ MNI coordinates $10,8,6 ; z$-score $=4.64 ; P<0.05$, FWE corrected, cluster-size 56 voxels; left: $-10,10,6$; $z$-score $=3.91$; $P<0.05$, FWE-corrected; cluster size 59). Only the groupby-stimulus interaction contrasting underweight against normalweight stimuli during the 'feel' task contributed to the overall significance. Neither did the 'weight' task show a significant effect for this interaction, nor did contrasting overweight against normal-weight stimuli show any for the feel or weight task (see Supplementary Information). To further compare the effects of task on the group-by-stimulus interaction for underweight and normal-weight stimuli, an appropriate F-test was used, setting the relevant contrast in task 'weight' in comparison to task 'feel', which produced a significant difference at the predefined level of significance of $P<0.05$ (FWE-corrected) in the ventral-striatal ROI (right: $8,10,6$; $z$-score $=3.56 ; P=0.013$; cluster size 26; left: $-10,12$, 6 ; $z$-score $=4.35 ; P=0.001$; cluster size 56 ) with the effect of the group-by-stimulus interaction higher for the 'feel' than for the 'weight' task. Therefore, further analysis of the group-by-stimulus interaction was constrained to the 'feel' task only, also because the effects of the different task types were not part of the main focus of the study.

Single-tailed $t$-contrasts testing revealed that relative to controls the significant group-by-stimulus interaction was driven by higher activity in patients upon processing underweight stimuli and by lower neural activity when processing normalweight images (Figure 2 and Table 2). The inverted $t$-contrast within the ROI did not yield significant effects $(P>0.05$, uncorrected). Post-hoc tests (directed $t$-contrasts; nominal level of $P<0.05$, uncorrected) on between-group differences for those voxels significant in the above single-tailed interaction $t$-contrast could also show that patients' increased neural activity upon underweight stimuli was significantly different from that of controls only in the left $(P<0.018)$ but not in the right ventral striatum $(P=0.075)$. In contrast, in both parts of the ventral striatum controls' neural activity upon normal-weight stimuli was significantly greater than that of patients (left: $P=0.002$; right: $P<0.001)$. Post-hoc testing on within-group differences showed that in the left $(P<0.001)$ and right $(P<0.001)$ ventral striatum controls showed significantly increased neural activity upon normal-weight stimuli compared to underweight stimuli. In patients neural activity upon both stimulus categories was alike in both parts of the ventral striatum (left: $P=0.480$; right: $P=0.417$; Figure 2).

At the predefined level of significance of $P<0.05$ (FWE corrected), the whole-brain analysis showed the right striatum
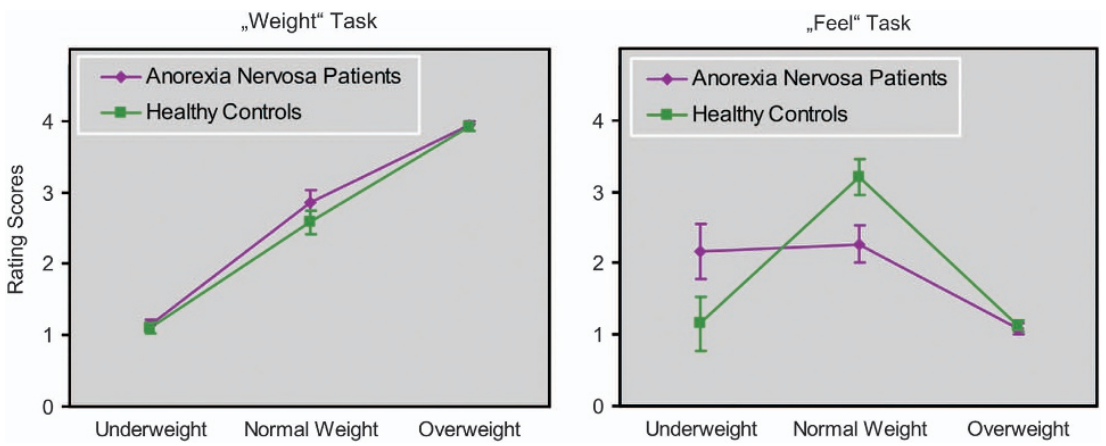

Figure 1. Average visual stimulus ratings ( $\pm 95 \%$ confidence interval) of young women with anorexia nervosa and healthy controls. In the 'weight' task, participants were asked to rate the weight of the woman depicted in the stimulus. In the 'feel' task, participants rated how they would feel if they were of the same body shape as depicted in the stimulus ( $1=$ very bad, $4=$ very good).
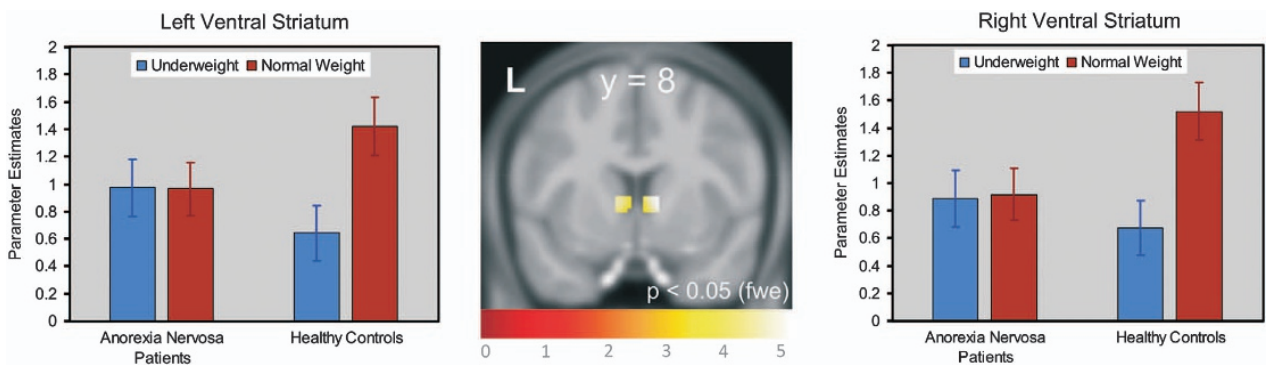

Figure 2. Significant $(P<0.05$, family-wise error-corrected) group-by-stimulus interaction for the 'feel' task in the left and right ventral striatum (see Table 2 for MNI coordinates of the left and right peak voxels and associated statistics). Bar charts show parameter estimates of blood oxygen level dependent responses averaged across significant voxels in young women with anorexia nervosa and healthy controls. Error is the $95 \%$ confidence interval. 
significant for the above single-tailed group-by-stimulus interaction contrast (peak voxel coordinates: $x=10, y=8, z=8$; $z$-score $=$ 4.89, $P=0.011$; cluster size $55(P=0.001)$; see Supplementary Information).

At a more lenient statistical threshold and within an extended ROI-based approach, the same group-by-stimulus interaction contrast was further explored. This analysis yielded effects in several brain regions, however with reduced effect sizes relative to those in the right striatum: bilateral anterior insula, putamen and right thalamus. This analysis also showed an effect in the midbrain comprising the substantia nigra/ventral tegmental area, with an effect size that was close below the effect size passing the strong, pre-defined statistical threshold of $P<0.05$, FWE-corrected (for more details, see Supplementary Information).

Comparison between young and adult AN patients

To evaluate the commonalities and differences between results obtained from adolescent and adult women with $\mathrm{AN}^{12}$ significant results from the 'feel' task in both samples are summarized in Figure 3. Adolescent and adult patients with AN rated

Table 2. Summary statistics of the significant group-by-stimulus (underweight, normal weight) interaction in ventral striatal $\mathrm{ROI}^{\mathrm{a}}$

\begin{tabular}{lccrrrr}
\hline Anatomical region & L/R & Cluster size & $\mathrm{x}$ & $\mathrm{y}$ & $\mathrm{z}$ & $\mathrm{z}$-score \\
\hline Ventral striatum & $\mathrm{R}$ & 97 & 10 & 8 & 6 & 4.89 \\
Ventral striatum & $\mathrm{L}$ & 97 & -10 & 10 & 4 & 4.44 \\
\hline
\end{tabular}

${ }^{a}$ Voxelwise significance of $P<0.05$, with family-wise error correction for multiple comparisons. underweight stimuli more positive and normal-weight stimuli less positive than the healthy subjects. In contrast to adult patients subjective ratings for underweight and normal-weight stimuli were alike in adolescent patients. The same pattern was evident in the fMRI data with greater neural activity upon underweight stimuli and lower neural activity upon normal-weight stimuli in both groups of patients relative to $\mathrm{HC}$ subjects. Again, the height of evoked neural activity upon both stimulus categories was alike in adolescent patients with AN in contrast to the adult patient group where neural activity associated with underweight stimuli was significantly greater than that associated with normal-weight stimuli.

\section{DISCUSSION}

Motivated by our previous results ${ }^{12}$ in adult patients with $A N$ demonstrating a dysfunctional signal in the ventral striatum as neural signature of maintenance, we investigated the pathogenesis of this dysfunctional signal shift in a group of adolescent patients with AN. The duration of illness in this group was about five times shorter than that of the adult sample. Compared to $\mathrm{HC}$ subjects the main effect of the relevant group-by-cue interaction was again located in the ventral striatum at almost the same location as it was for the adult group. However, the signal pattern differed from that of the adult group. While younger patients already demonstrated increased activation upon underweight stimuli and decreased activation upon normal-weight stimuli, there was no significant difference between both weight categories, which, by contrast, had been observed in the adult sample. Subjective ratings of underweight and normal-weight stimuli in a self-referential task showed the same pattern of results: Relative to controls an already increased positive rating of
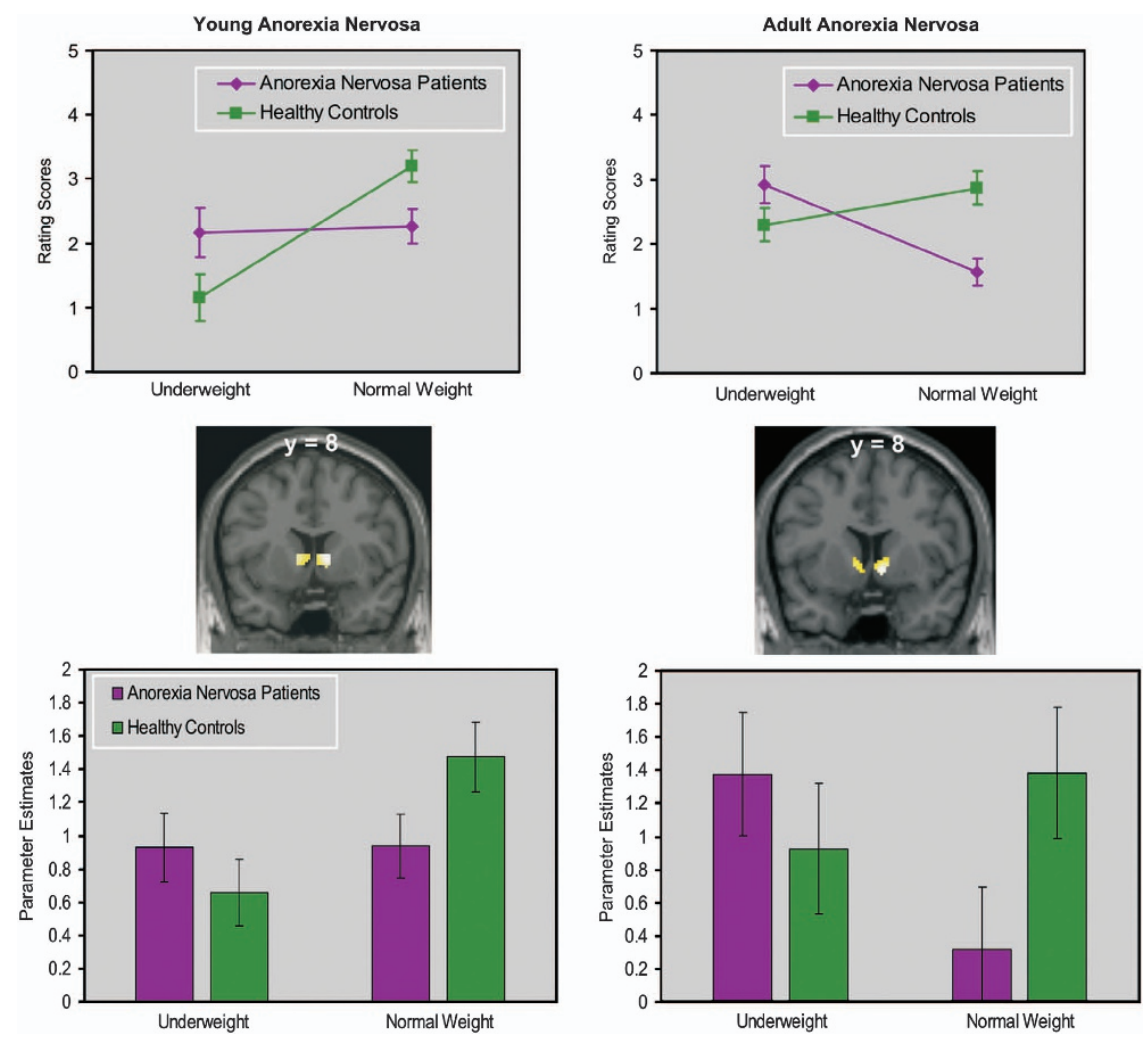

Figure 3. Comparison of significant results from the 'feel' task in young and adult patients with anorexia nervosa. Bar charts in the lower panels represent estimated evoked neural activity upon presentation of underweight and normal-weight stimuli averaged from the significant voxels in the left and right ventral striatum. Error is the $95 \%$ confidence interval. The lines in the upper panel represent group-averaged rating scores of underweight and normal-weight stimuli under the feel-task instruction. 
underweight and an already decreased positive rating of normal weight stimuli, but no difference between ratings for either stimulus category. This was also different to the adult group's ratings who had demonstrated a marked decrease of ratings from underweight to normal weight stimuli.

Present results strongly suggest that the ventral-striatal dysfunctional signal associated with maintenance of AN appears to evolve over time, which supports theories of AN as starvation dependence. . $^{3,11,12,20}$ These predict starvation in AN as conditioned response that develops over time by binding starvation associated cues to strong, dysfunctional motivational value by which the desire for food restriction is reinforced and perpetuates.

A putative mechanism for the conditioning process may be derived from animal studies on the interaction of stress and eating behaviour, which have shown that in response to stress, animals initially reduce caloric intake. This is likely to be induced by stressmediated activity changes of the hypothalamus-pituitary-adrenal axis (HPA) that can affect food intake through activation of the corticotropin-releasing factor (CRF) and its subsequent hypothalamus mediated effects on orexigenic neuropeptide hormonones. ${ }^{21-24}$ This initial occurrence of starvation can be bound to motivational salience due to an increase of dopamine (DA) release from dopaminergic midbrain neurons in the ventral tegmental area (VTA) known to project into the ventral striatum. ${ }^{3}$ This increase in DA can either be induced directly by stress-mediated CRF effects on CRF2 receptors of dopaminergic VTA neurons, or indirectly via hypothalamus-mediated effects of anorexigenic and orexigenic neuropeptide hormones on DA cells in the VTA. ${ }^{20,25-28}$ Recent data also support that CRF directly increases DA release in the dopaminergic reward system. ${ }^{29}$ Subsequently, this initial DA dependent binding of starvation to motivational salience may be re-instated by starvation alone since starvation per se has been shown to increase activity of the HPA-axis.,30 The resulting hypercortisolism triggers again an increase of DA from dopaminergic VTA neurons induced by the same mechanisms as described above: either directly by CRF effects or indirectly via effects of anorexigenic and orexigenic neuropeptide hormones. ${ }^{31}$ The more frequent this mechanism is repeated the more the ventral-striatal signalling is conditioned to starvation and associated cues. This might explain the development of signal shifts observed in our two samples of different duration of illness.

More recent data on the role of ATP-sensitive potassium (K-ATP) channels of dopaminergic midbrain neurons suggest a complementary mechanism to support this binding process. ${ }^{32}$ It was shown that NMDA receptor-dependent burst activity of DA neurons in the medial substantia nigra $(S N)$ is modulated by K-ATP channels. As these neurons project into the dorsal striatum burst activity was associated with exploratory behavior. When K-ATP channels were virally silenced burst activity and exploratory behaviour were reduced. Notably, the open probability of K-ATP channels is modulated by the metabolic status of the neuron and is increased under food restriction. ${ }^{33,34}$ Thus, starvation in AN could increase the open probability for K-ATP channels, thereby increasing the burst activity of SN/VTA neurons, resulting in an increased signalling of the ventral striatum that further supports the binding between starvation and motivational value.

Interestingly, the dependency of the open probability of K-ATP channels on the cells' metabolic status could also account for typical increases of hyperactivity that can be observed as AN progresses. This aligns with increases in physical exercise observed in activity-based animal models of AN where rodents perform excessive wheel running that in combination with reduced food intake finally leads to death. These extreme behavioral manifestations suggest that rodents become addicted to physical activity. Physical activity itself increases DA release in the reward system and changes DA binding. ${ }^{35}$ Furthermore, food anticipatory activity (FAA) can be observed in paradigms where food is available for a limited time period, that is, physical activity typically increases before food is delivered. ${ }^{36}$ FAA also appears in human patients with AN and involves the DA system, as administration of DA antagonists as well as lesions of DA neurons in the nucleus accumbens decreased FAA in the animal. ${ }^{20,37-39}$ Probably FAA also relies on changes in the open probability of K-ATP channels, as it should be highest when the metabolic status of the cells is lowest, that is, shortly before the meal when it is supplied at fixed intervals. At a larger scale, modulating the burst activity of DA midbrain cells by influencing their metabolic status may also account for the increase in physical exercise observed with progression of AN. If starvation alone is no longer sufficient to trigger motivational salient signalling, then an additional bout of DA signalling can be achieved by additionally lowering the metabolic status through increased exercise. This speculation aligns well with dose increasing in drug dependence.

The presented hypotheses on putative mechanisms target especially the two core symptoms of self-starvation and hyperactivity that reliably distinguish AN from other psychiatric disorders. ${ }^{3}$ Although disease-specific, we fully acknowledge that our suggestions are rather speculative and therefore await empirical evidence to a greater extent. ${ }^{11}$ Still, we hope our data will bring back AN's acknowledgement as a specific variant of a dependence disorder in the context of a conditioned shift in the responsiveness of the dopaminergic reward/motivation system. We believe that this perspective can motivate further promising research in the etiopathogenesis and therapy of this devastating disorder.

\section{CONFLICT OF INTEREST}

GG, A-KF, FS and KB declare that there are no conflicts of interest. US had research funding from FP7 programme (EU), got limited speaker's fees from Lilly Deutschland and from Pfizer, got a limited fee from Boehringer-Ingelheim for advisory activities concerning eating disorders, was involved in clinical trials with Janssen, Medice, Lilly Deutschland, Boehringer-Ingelheim and is currently working in a clinical trial related to Lundbeck.

\section{REFERENCES}

1 Schoemaker C. Does early intervention improve the prognosis in anorexia nervosa? A systematic review of the treatment-outcome literature. Int J Eating Disorders 1997; 21: 1-15.

2 Eddy KT, Le Grange D, Crosby RD, Hoste RR, Doyle AC, Smyth A et al. Diagnostic classification of eating disorders in children and adolescents: how does DSM-IV-TR compare to empirically-derived categories? J Am Acad Child Adolesc Psychiatry 2010; 49: 277-287, quiz 293.

3 Bergh C, Sodersten P. Anorexia nervosa, self-starvation and the reward of stress. Nat Med 1996; 2: 21-22.

4 Berridge KC. The debate over dopamine's role in reward: the case for incentive salience. Psychopharmacology 2007; 191: 391-431.

5 Hollerman JR, Tremblay L, Schultz W. Involvement of basal ganglia and orbitofrontal cortex in goal-directed behavior. Prog Brain Res 2000; 126: 193-215.

6 Jensen J, Walter H. Incentive motivational salience and the human brain. Restor Neurol Neurosci advance online publication, 29 March 2013; doi:10.3233/RNN139006 (e-pub ahead of print).

7 Koob GF, Le Moal M. Review. Neurobiological mechanisms for opponent motivational processes in addiction. Philos Trans $R$ Soc Lond B Biol Sci 2008; 363: 3113-3123.

8 Robbins TW, Everitt BJ. Neurobehavioural mechanisms of reward and motivation. Curr Opin Neurobiol 1996; 6: 228-236.

9 Zink CF, Pagnoni G, Chappelow J, Martin-Skurski M, Berns GS. Human striatal activation reflects degree of stimulus saliency. Neuroimage 2006; 29: 977-983.

10 Kim SF. Animal models of eating disorders. Neuroscience 2012; 211: 2-12.

11 Zink CF, Weinberger DR. Cracking the moody brain: the rewards of self starvation. Nat Med 2010; 16: 1382-1383.

12 Fladung AK, Gron G, Grammer K, Herrnberger B, Schilly E, Grasteit S et al. A neural signature of anorexia nervosa in the ventral striatal reward system. Am J Psychiatry 2010; 167: 206-212.

13 Association AP. Diagnostic and Statistical Manual of Mental Disorders: DSM-IV vol. 4. American Psychiatric Press: Washington, DC, 1994. 
14 Kromeyer-Hauschild K, Wabitsch M, Kunze D, Geller F, Geiß HC, Hesse V et al. Perzentile für den Body-mass-Index für das Kindes- und Jugendalter unter Heranziehung verschiedener deutscher Stichproben. Monatsschr Kinderheilkunde 2001; 149: 807-818.

15 Garner DM. Eating Disorder Inventory-2 (Professional Manual). Psychological Assessment Ressources Inc.: Odessa, FL, 1991.

16 Fairburn CG, Beglin SJ. Assessment of eating disorders: interview or self-report questionnaire? Int J Eating Disord 1994; 16: 363-370.

17 Ashburner J. A fast diffeomorphic image registration algorithm. Neuroimage 2007; 38: 95-113.

18 Maldjian JA, Laurienti PJ, Burdette JH. Precentral gyrus discrepancy in electronic versions of the Talairach Atlas. Neuroimage 2004; 21(1): 450-455.

19 Maldjian JA, Laurienti PJ, Kraft RA, Burdette JH. An automated method for neuroanatomic and cytoarchitectonic atlas-based interrogation of $\mathrm{FMRI}$ data sets. Neuroimage 2003; 19: 1233-1239.

20 Scheurink AJ, Boersma GJ, Nergardh R, Sodersten P. Neurobiology of hyperactivity and reward: agreeable restlessness in anorexia nervosa. Physiol Behav 2010; 100: 490-495.

21 Jahng JW. An animal model of eating disorders associated with stressful experience in early life. Hormones Behav 2011; 59: 213-220.

22 Lo Sauro C, Ravaldi C, Cabras PL, Faravelli C, Stress Ricca V. Hypothalamicpituitary-adrenal axis and eating disorders. Neuropsychobiology 2008; 57: 95-115.

23 Dunn AJ, Berridge CW. Is corticotropin-releasing factor a mediator of stress responses? Ann NY Acad Sci 1990; 579: 183-191.

24 Saegusa Y, Takeda H, Muto S, Nakagawa K, Ohnishi S, Sadakane C et al. Decreased plasma ghrelin contributes to anorexia following novelty stress. Am J Physiol Endocrinol Metab 2011; 301: E685-E696.

25 Wang B, You ZB, Rice KC, Wise RA. Stress-induced relapse to cocaine seeking: roles for the CRF(2) receptor and CRF-binding protein in the ventral tegmental area of the rat. Psychopharmacology 2007; 193: 283-294.

26 Connan F, Campbell IC, Katzman M, Lightman SL, Treasure J. A neurodevelopmental model for anorexia nervosa. Physiol Behav 2003; 79: 13-24.

27 Skibicka KP, Hansson C, Alvarez-Crespo M, Friberg PA, Dickson SL. Ghrelin directly targets the ventral tegmental area to increase food motivation. Neuroscience 2011; 180: 129-137.

28 van Zessen R, van der Plasse G, Adan RA. Contribution of the mesolimbic dopamine system in mediating the effects of leptin and ghrelin on feeding. Proc Nutr Soc 2012; 71: 435-445.
29 Lemos JC, Wanat MJ, Smith JS, Reyes BA, Hollon NG, Van Bockstaele EJ et al. Severe stress switches CRF action in the nucleus accumbens from appetitive to aversive. Nature 2012; 490: 402-406.

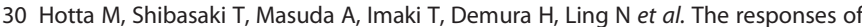
plasma adrenocorticotropin and cortisol to corticotropin-releasing hormone $(\mathrm{CRH})$ and cerebrospinal fluid immunoreactive $\mathrm{CRH}$ in anorexia nervosa patients. J Clin Endocrinol Metab 1986; 62: 319-324.

31 Piazza PV, Rouge-Pont F, Deroche V, Maccari S, Simon H, Le Moal M. Glucocorticoids have state-dependent stimulant effects on the mesencephalic dopaminergic transmission. Proc Natl Acad Sci USA 1996; 93 8716-8720.

32 Schiemann J, Schlaudraff F, Klose V, Bingmer M, Seino S, Magill PJ et al. K-ATP channels in dopamine substantia nigra neurons control bursting and noveltyinduced exploration. Nature Neuroscience 2012; 15: 1272-1280.

33 Liss B, Roeper J. ATP-sensitive potassium channels in dopaminergic neurons: transducers of mitochondrial dysfunction. News Physiol Sci 2001; 16: 214-217.

34 Kishore P, Boucai L, Zhang K, Li W, Koppaka S, Kehlenbrink S et al. Activation of K (ATP) channels suppresses glucose production in humans. $J$ Clin Invest 2011; 121 4916-4920.

35 Foley TE, Fleshner M. Neuroplasticity of dopamine circuits after exercise: implications for central fatigue. Neuromol Med 2008; 10: 67-80.

36 Moran TH, Tamashiro KL. Curt Richter: spontaneous activity and food intake. Appetite 2007; 49: 368-375.

37 Bruch H. Perceptual and conceptual disturbances in anorexia nervosa. Psychosom Med 1962; 24: 187-194.

38 Kelley AE, Baldo BA, Pratt WE. A proposed hypothalamic-thalamic-striatal axis for the integration of energy balance, arousal, and food reward. J Comp Neurol 2005 493: 72-85.

39 Verhagen LA, Luijendijk MC, Hillebrand JJ, Adan RA. Dopamine antagonism inhibits anorectic behavior in an animal model for anorexia nervosa. Eur Neuropsychopharmacol 2009; 19: 153-160.

This work is licensed under a Creative Commons AttributionNonCommercial-ShareAlike 3.0 Unported License. To view a copy of this license, visit http://creativecommons.org/licenses/by-nc-sa/3.0/

Supplementary Information accompanies the paper on the Translational Psychiatry website (http://www.nature.com/tp) 